\title{
Single-Crystal Silicon HARPSS Capacitive Resonators with Submicron Gap-Spacing
}

\author{
Seong Yoel No, Akinori Hashimura, Siavash Pourkamali, and Farrokh Ayazi \\ School of Electrical and Computer Engineering \\ Georgia Institute of Technology, GA 30332-0250 \\ E-mail: ayazi@ece.gatech.edu, Tel: (404) 894-9496, Fax: (404) 894-4700
}

\begin{abstract}
This paper reports on the fabrication and characterization of single crystal silicon (SCS) in-plane capacitive resonators with submicron gap spacing using the HARPSS process. The resonating element is made out of single crystal silicon and the drive and sense electrodes are made out of trench-refilled polysilicon, hence yielding an all-silicon microresonator with potentially better temperature and long-term stability. The capacitive gap is defined by a sacrificial oxide layer, which can be potentially reduced to the ten's of nanometer range $(700 \mathrm{~nm}$ reported in this paper). Quality factor as high as 67,000 was measured in $1 \mathrm{mT}$ Torr vacuum for the $40 \mathrm{kHz}$ fundamental resonance frequency of a clamped-clamped beam SCS HARPSS resonator. A $510 \mu \mathrm{m}$ long, $5.5 \mu \mathrm{m}$ wide, $20 \mu \mathrm{m}$ thick clamped-clamped beam resonator was operated in its third resonance mode at $1 \mathrm{MHz}$ and demonstrated a $Q$ of 11,000 under vacuum. Electrostatic tuning characteristics of the resonators have been measured and reported.
\end{abstract}

\section{INTRODUCTION}

MEMS resonators are a potential candidate to replace current off-chip frequency selective mechanical components such as crystal, ceramic and SAW devices in wireless communication systems. High quality factors, small size and compatibility with IC integration are the major advantages of the silicon MEMS resonators over their counterparts. Extension of the frequency range of capacitive MEMS resonators into the low $\mathrm{GHz}$ range requires process technologies that can yield 10-100 nanometer capacitive gap spacings in between the high quality factor (Q) resonating structure and the drive and sense electrodes [1].

A number of poly and single crystalline silicon resonators have been reported in literature [2-7]. Polysilicon capacitive resonators with submicron gap spacing have been demonstrated with metal electrodes that result in a thermal mismatch [2]. Single crystal silicon (SCS) is a more attractive structural material for microresonators compared to polysilicon due to its inherent high mechanical quality factor and independence from various process parameters. However, single crystal silicon resonators reported previously in literature had either complex non-capacitive sense and drive mechanisms [4], large capacitive gaps [5], or low quality factor [6]; such issues may compromise design flexibility and limit the high frequency applications of the microresonators.

This paper reports, for the first time, the implementation of high-Q single crystal silicon resonators with submicron gap spacing using an enhanced and modified version of the HARPSS process. The in-plane resonating element is made out of single crystal silicon and the drive and sense electrodes are made out of trench-refilled polysilicon, hence yielding an all-silicon microresonator. Measurement results on the $\mathrm{Q}$ and tuning characteristics of the fabricated beam resonators in the fundamental and third order modes are presented.

\section{RESONATOR FABRICATION}

The HARPSS process [7] has been previously used to make thick polysilicon resonators $[\mathbf{7 , 8}]$. In this paper, the HARPSS process is modified to implement single crystal silicon resonating structures with trench-refilled poly electrodes. The schematic diagram of an in-plane clamped-clamped beam resonator fabricated through the HARPSS process is shown in Fig. 1.

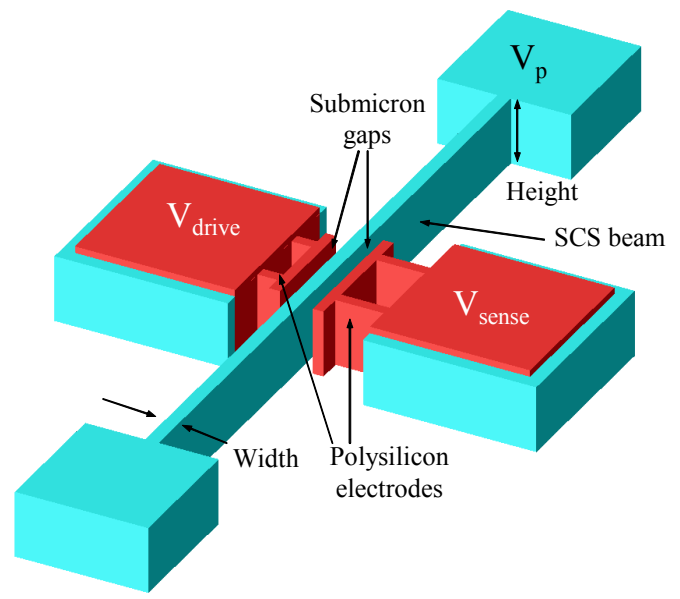

Figure 1. HARPSS clamped-clamped beam SCS resonator

The fabrication process flow is shown in Fig. 2. The SCS resonating beam is defined by two adjacent high aspect-ratio trenches that are 2 to $5 \mu \mathrm{m}$ wide and up to $100 \mu \mathrm{m}$ deep. The trenches are etched in an inductively-coupled plasma (ICP) deep reactive ion etching (DRIE) system using the Bosch process. The height of the trenches determines the height of the resonator. A thin conformal layer of sacrificial LPCVD high-temperature oxide is then deposited and trenches are consequently refilled with doped LPCVD poly to form the vertical electrodes. The lateral gap spacing is defined by the thickness of the deposited oxide layer, and thus can be scaled down to the ten's of nanometer range [1].

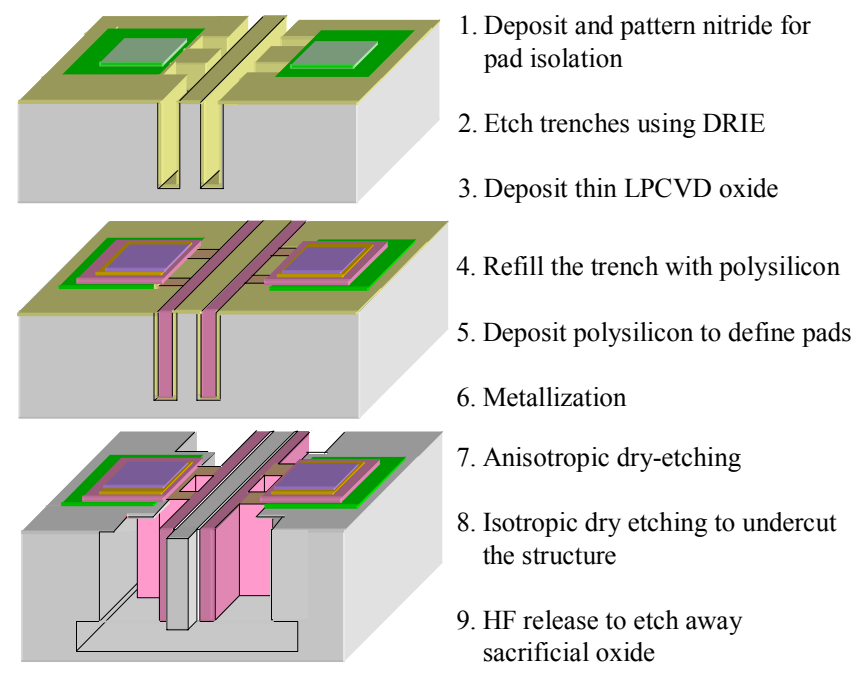

Figure 2. Process flow for HARPSS single crystal silicon beam resonators. 
The resonator is released from the silicon substrate using a dry silicon etch in $\mathrm{SF}_{6}$ plasma, consisting of an anisotropic followed by an isotropic etch to undercut the structures. Finally, the sacrificial oxide is removed in a $\mathrm{HF}: \mathrm{H}_{2} \mathrm{O}(1: 1)$ solution. Fig. 3 shows the SEM picture of a fabricated clamped-clamped beam resonator with a length of $510 \mu \mathrm{m}$, width of $6.5 \mu \mathrm{m}$, height of $20 \mu \mathrm{m}$, and a capacitive gap of 700nm. Figures 4 and 5 are the SEM pictures of the beam electrodes area, showing the single crystal beam, the polysilicon electrodes and the submicron gap.

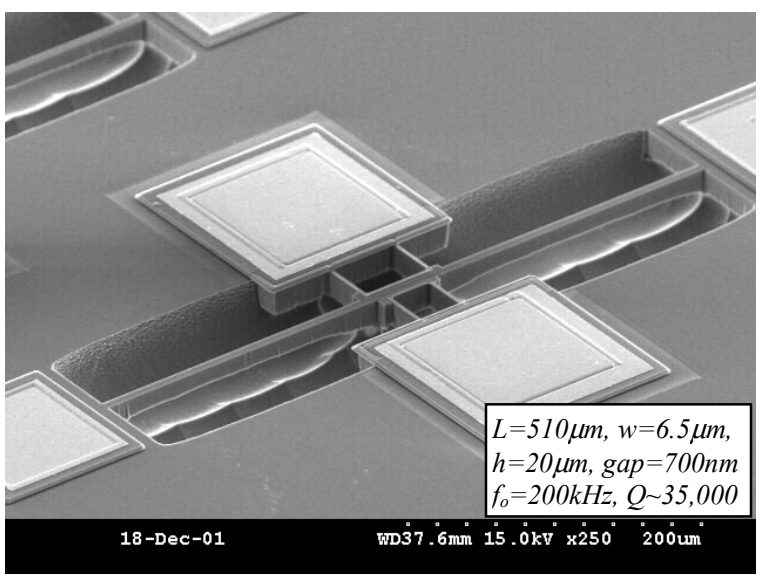

Figure 3. A $510 \mu \mathrm{m}$ long, $6.5 \mu \mathrm{m}$ wide, $20 \mu \mathrm{m}$ tall clamped-clamped beam HARPSS SCS resonator with a gap spacing of $700 \mathrm{~nm}$.

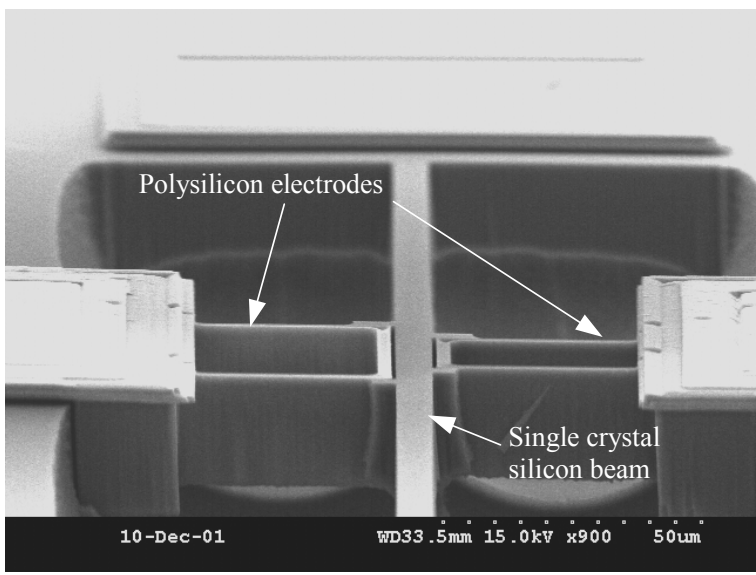

Figure 4. SEM of the electrode area showing the polysilicon electrodes as tall as the beam separated by submicron gaps.

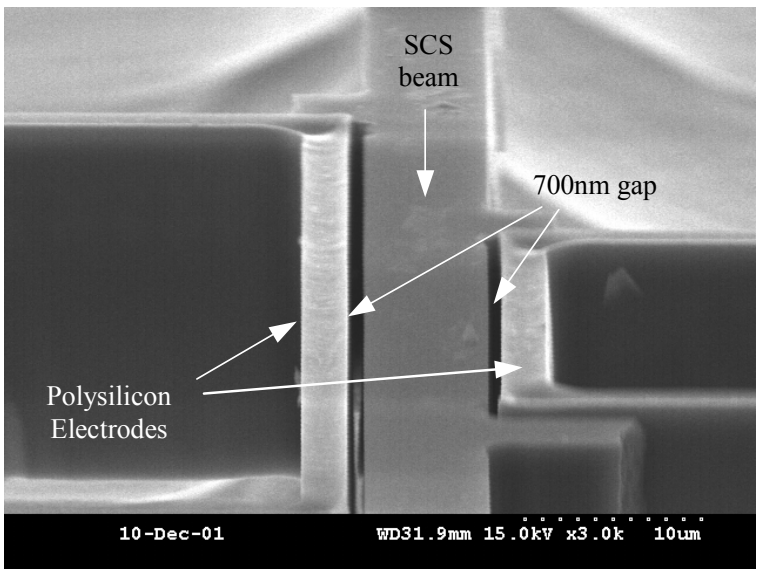

Figure 5. Close-up view of the electrode area showing the $700 \mathrm{~nm}$ gap spacing.
Disk resonators have also been fabricated using the above process to obtain higher resonance frequency. The SEM picture of a $20 \mu \mathrm{m}$ diameter SCS disk resonator fabricated using the HARPSS process is shown in Fig. 6. Characterization and measurement results for the disk resonators will be reported in the future.

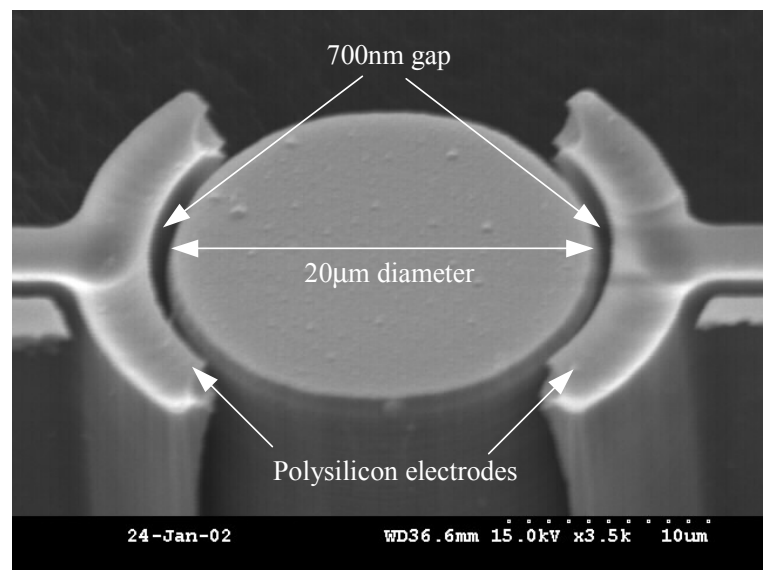

Figure 6. SEM picture of a $20 \mu \mathrm{m}$ in diameter SCS disk resonator with polysilicon electrodes.

In this process, the trenches define: 1) the boundary of the resonating structure, and 2) the polysilicon electrodes. At the end of the process, all the polysilicon inside the trenches should be removed, except for the electrode area. Two methods were investigated, as shown in Fig. 7(a) and (b), to separate the polysilicon in the electrode area from the areas that only define the boundary of the resonator. The first method is to physically disconnect the polysilicon electrode by using discontinuous trenches and etch away the polysilicon inside the trenches that define the boundary of the resonator. However, this method creates "stubs" on the sides of the beam as shown in the SEM of Fig. 7(a), introducing residual extra mass. FEM simulations using ANSYS have shown that these stubs do not alter the resonance frequency significantly. The size of these stubs can be controlled by characterizing the isotropic release etch. The alternative method is to use a continuous trench to define the boundary of the resonator and reduce the width of the trench in certain areas. As shown in Fig. 7(b), the narrow trench (located in between the regular size trenches) is primarily filled by the sacrificial oxide.

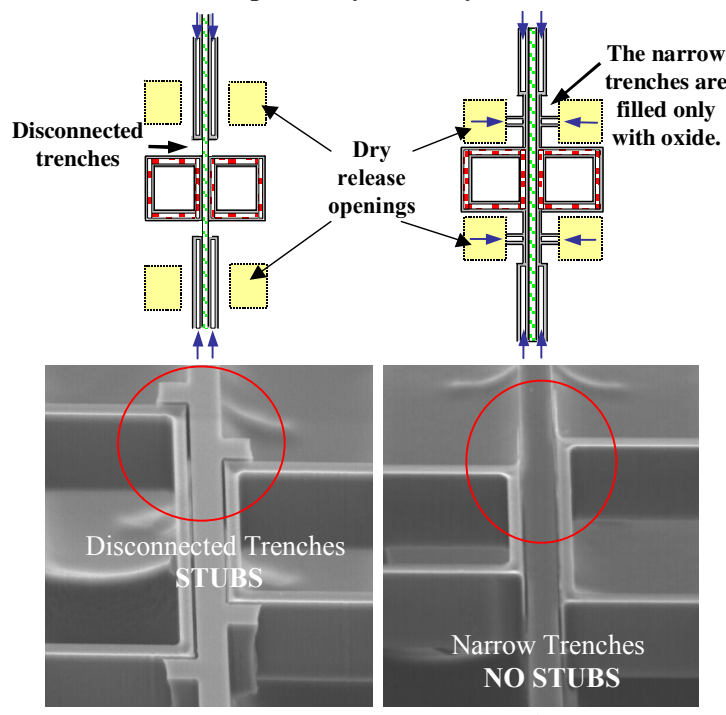

(a)

(b)

Figure 7. Diagram and SEM of the separation methods using a) disconnected trenches, b) narrow trenches. 
Due to the presence of voids in the middle of oxide-filled narrow trenches, a thin residual poly line may be formed, connecting the electrode trench-refilled poly to the boundary poly. This parasitic poly will be subsequently etched away during the isotropic etch step of the release process.

\section{MEASUREMENT RESULTS}

Prototypes of the fabricated SCS beam resonators were tested under vacuum in a two-port configuration using an Agilent 4395A network analyzer. A low noise JFET source-follower with a gain stage was used to interface with the resonators. The sensing interface circuit was built on a printed circuit board (PCB) using surface mount components and the MEMS resonator chip was mounted on the board and wire-bonded. The PCB was placed in a custom vacuum system, which kept the pressure less than 1mTorr.

Fig. 8 shows the plot of a typical frequency response taken from the network analyzer, showing a Q of 62,000 at $49 \mathrm{kHz}$ for a $1100 \mu \mathrm{m}$ long, $7.5 \mu \mathrm{m}$ wide and $20 \mu \mathrm{m}$ tall SCS clamped-clamped beam resonator under $1 \mathrm{mT}$ Torr vacuum. The summary of the measured $\mathrm{Q}$ values for the beam resonators with fundamental frequencies ranging from $41.5 \mathrm{kHz}$ to $530 \mathrm{kHz}$ is given in Fig. 9. A $\mathrm{Q}$ of $\sim 20,000$ was measured for the $0.5 \mathrm{MHz}$ beam resonator.

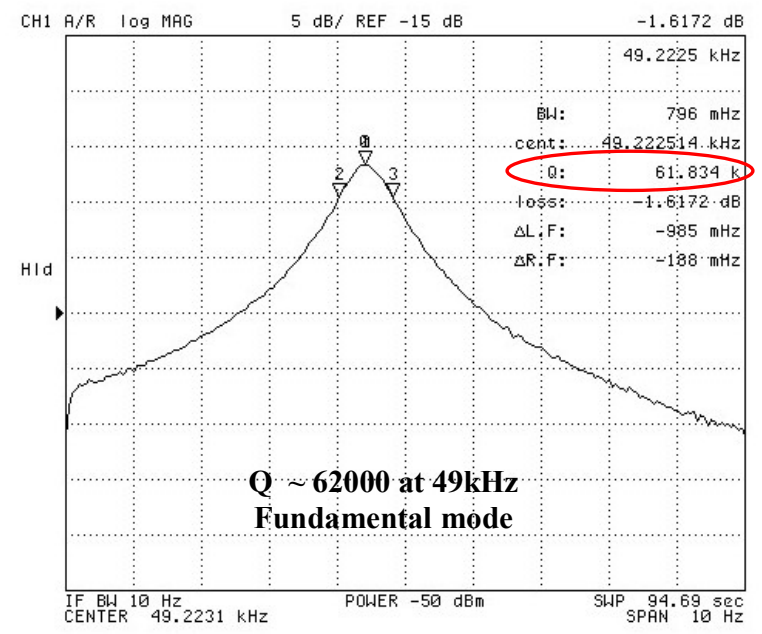

Figure 8. Response of a $1100 \mu \mathrm{m}$ long, $7.5 \mu \mathrm{m}$ wide, $20 \mu \mathrm{m}$ tall HARPSS SCS beam resonator, showing a $Q$ of $\sim 62000$ at $49 \mathrm{kHz}$.

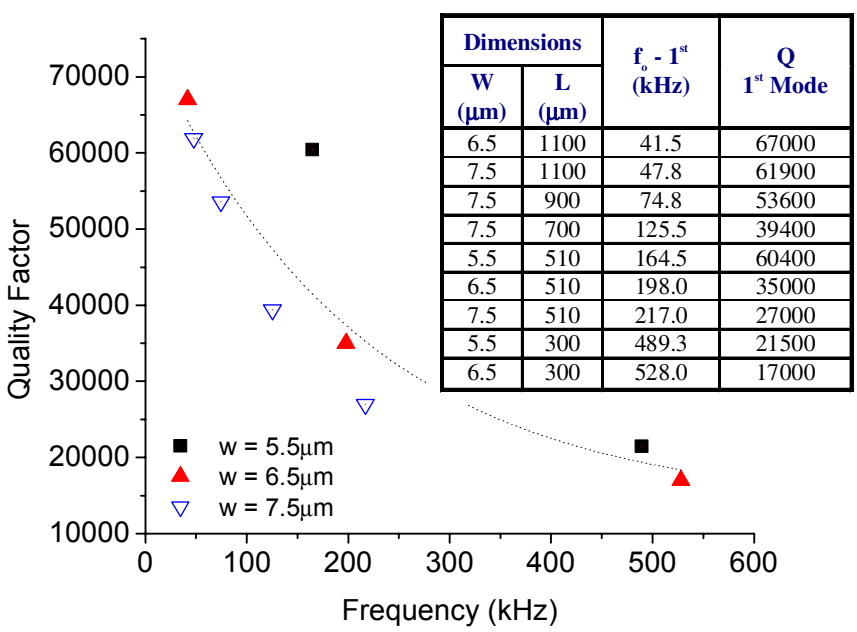

Figure 9. Plot of measured $Q$ vs. measured fundamental resonance frequencies of SCS beams. Q decreases with increasing frequency.
Fig. 10 shows the comparison between the measured and theoretical frequency tuning characteristics for a $300 \mu \mathrm{m}$ long, $6.5 \mu \mathrm{m}$ wide resonator by changing the DC polarization voltage $(V p)$. The mismatch is mainly due to fabrication uncertainties in defining the dimensions of the structure, which alter the overall stiffness. The resonance frequency changed from $505 \mathrm{kHz}$ to $450 \mathrm{kHz}$ by changing the polarization voltage by more than $40 \mathrm{~V}$ (the calculated pull-in voltage was $56 \mathrm{~V}$ ), providing a large electrostatic tuning range $(\sim 10 \%)$.

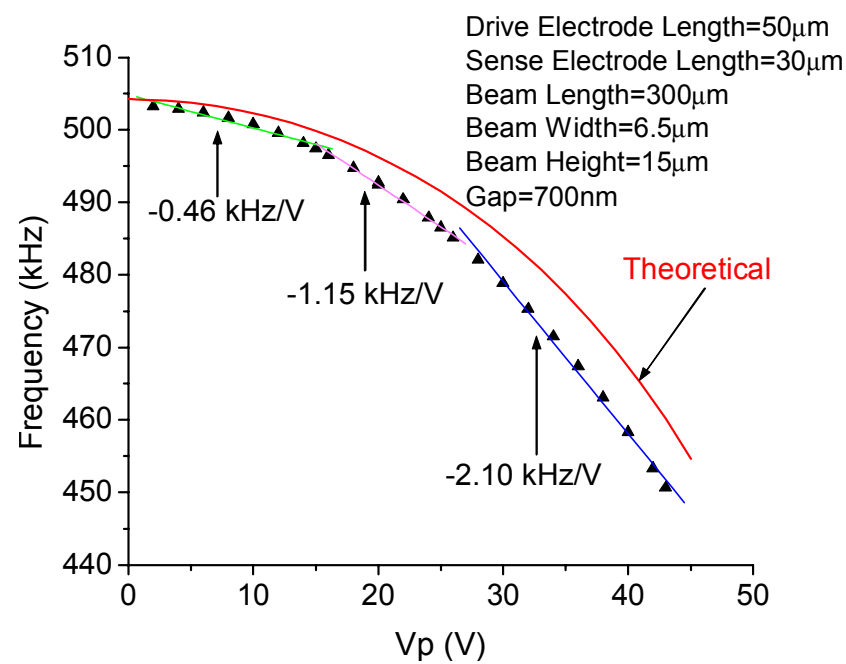

Figure 10. Plot of the resonance frequency vs. polarization voltage for a $300 \mu \mathrm{m}$ long, $6.5 \mu \mathrm{m}$ wide beam resonator.

\subsection{Third flexural resonance mode}

In order to achieve higher operating frequencies, a few of the clamped-clamped beam resonators were actuated in their third flexural mode and the quality factors were measured. The second mode could not be actuated due to the midway position of the drive electrode. A Q of 10,700 was measured at $1.03 \mathrm{MHz}$ for the third resonance mode of a $510 \mu \mathrm{m}$ long, $5.5 \mu \mathrm{m}$ wide SCS beam resonator (the $1^{\text {st }}$ mode is at $165 \mathrm{kHz}$ ). Figure 11 shows the measured resonance peak for this mode (the anti-resonance peak is due to the parasitic feedthrough capacitance), and provides a summary of the Q measurement results for the $3^{\text {rd }}$ mode of the beam resonators with other dimensions.

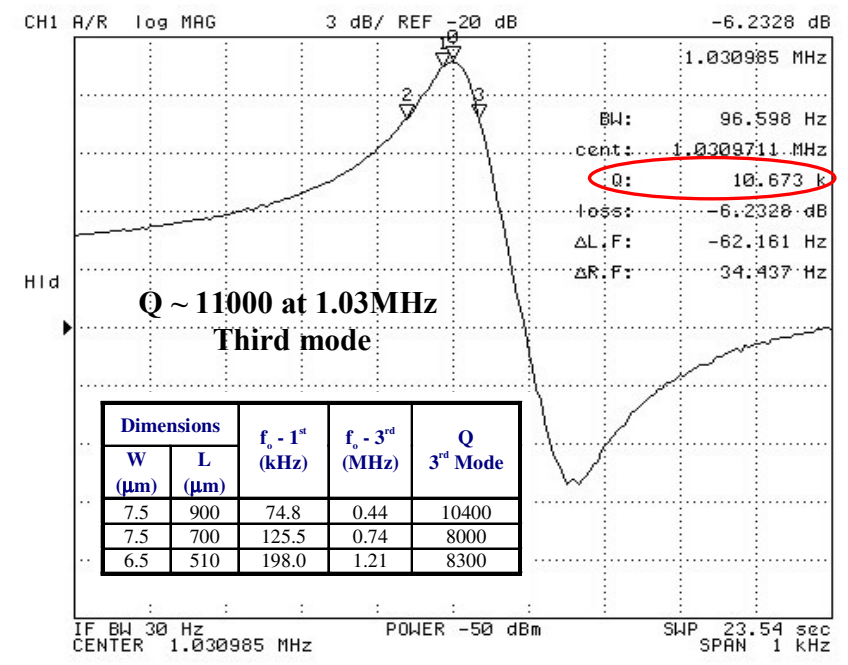

Figure 11. Plot of $Q(\sim 10,700)$ for a clamped-clamped SCS beam resonator operating in its $3^{\text {rd }}$ flexural mode at $1 \mathrm{MHz}$. 


\subsection{Loss Mechanisms}

Several possible loss mechanisms have been investigated by researchers including the thermoelastic damping (TED), support, and surface related loss mechanisms [5,9-11]. Using the TED relationships for beam resonators reported in references [9] and [10], a minimum $Q$ of 11,000 occurs at frequencies of $4.8,3.3$ and $2.1 \mathrm{MHz}$ for SCS beam widths of $5.5,6.5$, and $7.5 \mu \mathrm{m}$, respectively. Figure 12 shows the plots of calculated $\mathrm{Q}_{\mathrm{TED}}$ along with the $\mathrm{Q}$ measurement results for $5.5 \mu \mathrm{m}$ and $7.5 \mu \mathrm{m}$ wide SCS beam resonators. Although the measured $\mathrm{Q}$ values for each beam-width seem to follow the trend predicted by the TED loss ( $Q$ decreases with increasing frequency), there is a substantial offset associated with the measured $Q$ values (a factor of 2-3 less than $Q_{T E D}$ ).

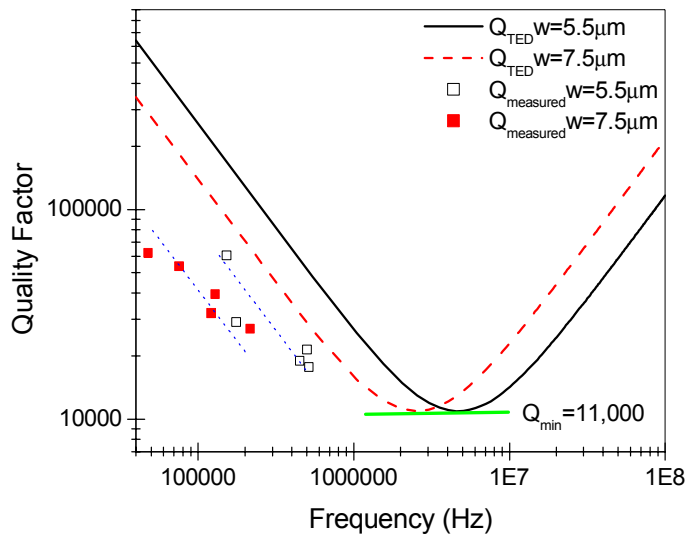

Figure 12. $Q$ vs. frequency showing the measured quality factor and calculated $Q_{T E D}$ for beam-widths of $5.5 \mu \mathrm{m}$ and $7.5 \mu \mathrm{m}$.

The observed offset cannot be due to excessive support loss. For the beam resonators, the support loss is proportional to the third power of the length to width ratio $(l / w)^{3}[\mathbf{1 1}]$. The length to width ratios are greater than $40(l / w>40)$ for all the beam resonators studied in this paper, suggesting much larger $\mathrm{Q}_{\text {sur }}$ values than what was measured. However, the surface loss could very well be a major source of dissipation for the resonators reported here. Figure 13 shows the sidewall of a prototype beam resonator, showing significant surface roughness that is indeed present in all of the beam resonators due to the un-optimized DRIE process used in fabrication. The DRIE process can be easily optimized to eliminate the striation on the sidewall and increase the Q. As reported in reference [5], the losses associated with surface roughness can be significantly reduced by growing a thin oxide and subsequently removing it.

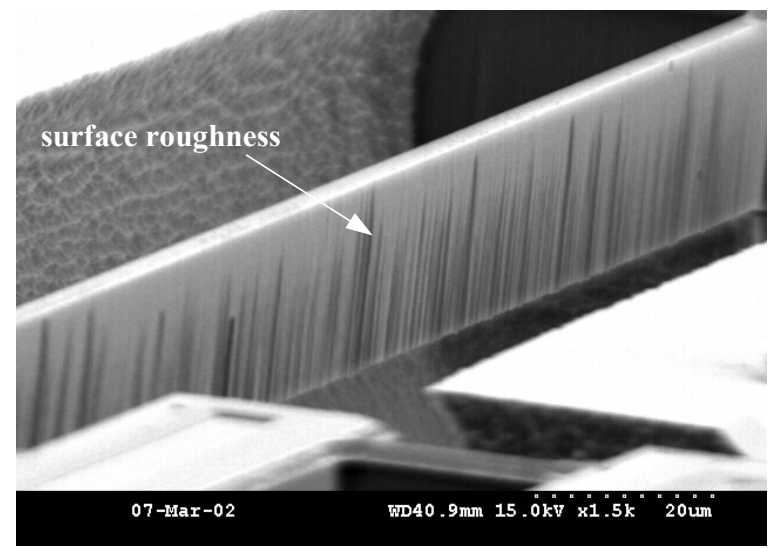

Figure 13. SEM of the sidewall of a SCS resonator. The vertical "striation" is caused by unoptimized DRIE etch conditions.

\section{CONCLUSIONS}

All-silicon high-Q capacitive micromechanical resonators with submicron gap-spacing utilizing single crystal silicon as the resonating element and polysilicon as the electrodes have been reported. The resonators were fabricated using the HARPSS process and uniform capacitive gaps of $0.7 \mu \mathrm{m}$ are demonstrated. Quality factors as high as 67,000 for the $1^{\text {st }}$ mode at $40 \mathrm{kHz}$, and 11,000 for the $3^{\text {rd }}$ mode at $1 \mathrm{MHz}$ have been measured for clampedclamped SCS beam resonators. Investigation of various loss mechanisms revealed that the measured quality factor follows the behavior predicted by the TED loss mechanism with an offset. The reason for the offset is believed to be due to the excessive surface roughness of the SCS beam. Optimization of the DRIE process is expected to reduce the surface roughness and increase the Q. Future work will report on confirmation of the above as well as characterization of the UHF SCS HARPSS resonators.

\section{Acknowledgements}

This work was supported by DARPA under contract \# DAAH0101-1-R004. Authors would like to thank Gavin Ho for providing the 3D diagram as well as discussion on TED loss, and the staff at the Georgia Tech Microelectronics Research Center for their assistance.

\section{REFERENCES}

1. S. Y. No and F. Ayazi, "The HARPSS Process for Fabrication of Nano-Precision Silicon Electromechanical Resonators", IEEE Conf. on Nanotechnology, 10/28-30/01, (2001), pp.489-494.

2. W.-T. Hsu, et al., "Q-Optimized Lateral Free-Free Beam Micromechanical Resonators", Int. Conf. On Solid-State Sensors and Actuators (Transducer'01), Munich, Germany, 6/10-14/01, (2001), pp. 1110-1113.

3. C. Bourgeois et al., "Design of Resonators for the Determination of the Temperature Coefficients of Elastic Constants of Monocrystalline Silicon", 1997 IEEE Int. Freq. Control Symp., Orlando, Fl. 5/28-30/97, (1997) pp.791-799.

4. K. Petersen, et al., "Resonant Beam Pressure Sensor Fabricated with Silicon Fusion Bonding", $6^{\text {th }}$ Int. Conf. on Solid State Sensors and Actuators, (Tranducer'91), San Francisco, CA, 6/24-28/91, (1991), pp.664-667.

5. R. E. Mihailovich, and N. C. MacDonald, "Dissipation measurements of vacuum-operated single-crystal silicon microresonators", Sensors and Actuators A ,50, 199, (1995).

6. T. Mattila et al. " $14 \mathrm{MHz}$ Micromechanical Oscilaltor", $11^{\text {th }}$ Int. Conf. on Solid-State Sensors and Actuators, (Transducer'01), Munich, Germany, 6/10-14/01, (2001), pp.1102-1105.

7. F. Ayazi and K. Najafi, "High Aspect-Ratio Combined Poly and Single-Crystal Silicon (HARPSS) MEMS Technology", JMEMS, 9(3), 288, (2000).

8. F. Ayazi and K. Najafi, "A HARPSS Polysilicon Vibrating Ring Gyroscope," JMEMS, June 2001, pp. 169-179.

9. T. V. Roszhart, "The Effect of Thermoelastic Internal Friction on the Q of Micromachined Silicon Resonators”, IEEE Solid State Sensor and Actuator Workshop, Hilton Head, SC, 6/4-7/90, (1990), pp.489-494.

10. R. Lifshitz and M. L. Roukes, "Theromoelastic damping in micro- and nanomechanical systems", Phy. Rev. B., 61(8), 5600, (2000).

11. K. Y. Yasumura et al., "Quality Factors in Micron- and Submicron-Thick Cantilevers", JMEMS, 9(1), 117, (2000). 